\title{
Experimental Recovery from Anemia in Japanese Flounder Challenged with the Monogenean Neoheterobothrium hirame
}

\author{
Tomoyoshi Yoshinaga ${ }^{1 *}$, Takashi Kamaishi ${ }^{1}$, Hanako Ikeda $^{2}$ and Minoru Sorimachi ${ }^{1}$ \\ ${ }^{1}$ National Research Institute of Aquaculture, Fisheries Research Agency, \\ Nansei, Mie 516-0193, Japan \\ ${ }^{2}$ Fukui Prefectural Fisheries Experimental Station, Tsuruga, \\ Fukui 914-0843, Japan
}

(Received April 23, 2001)

\begin{abstract}
For clarification of the etiological agent of the anemia recently prevailing in wild and cultured populations of the Japanese flounder Paralichthys olivaceus, flounder were challenged with the monogenean Neoheterobothrium hirame. In the challenged flounder, the hematological characteristics similar to those in the recent anemia were induced. Moreover, when $N$. hirame were artificially removed from the anemic flounder produced by the challenge, the hematological conditions recovered to normal levels. These results suggest that the parasite itself can induce anemia in Japanese flounder and refute a possibility that the parasite acts as a vector of other pathogens. They also indicate that the removal of $N$. hirame from anemic flounder is effective as a treatment of the anemia.
\end{abstract}

Key words: Neoheterobothrium hirame, anemia, Paralichthys olivaceus, Monogenea, Japanese flounder

Recently, anemia has been widespread in both wild and cultured populations of the Japanese flounder Paralichthys olivaceus in Japan. The anemia is characterized hematologically by a low hemoglobin concentration $(\mathrm{Hb})$, the mass appearance of immature erythrocytes and abnormal erythrocytes having a vacuolated or weakly stained cytoplasm and histologically by the absence of clear lesions (Miwa and Inouye, 1999; Michine, 1999; Yoshinaga et al., 2000a). Although the causative agent of the anemia has not been fully clarified yet, several studies have been carried out to determine the cause. We previously reported that anemic flounder had been infected with the monogenean parasite Neoheterobothrium hirame at high prevalences (Yoshinaga et al., 2000a) and that the hematological characteristics similar to those of the recent anemia were generated by challenging Japanese flounder with the parasite (Yoshinaga et al., 2001), suggesting that the parasite is the cause of the anemia. On the other hand, Miwa and Inouye (1999) observed virus-like particles in cells in the hematopoietic tissues in the kidney and

* Corresponding author

E-mail: crypto@fra.affrc.go.jp spleen of anemic flounder and suggested that a virus causes the anemia and $N$. hirame may play a role as a vector of the causative virus.

This study was conducted to clarify whether $N$. hirame is involved in the anemia in Japanese flounder as a vector of some causative virus or the parasite itself can generate anemic conditions in Japanese flounder. For this purpose, we produced anemic flounder by a challenge with $N$. hirame and removed the parasite from them, assuming that the anemia would not be cured only by the removal of the parasite, if the parasite worked as a vector of the causative virus.

\section{Materials and Methods}

Juvenile Japanese flounder were purchased from a hatchery where Neoheterobothrium hirame infection had not been recorded. The flounder were maintained at the National Research Institute of Aquaculture (NRIA), Mie, Japan, until used. Wild anemic flounder infected with $N$. hirame were captured in Wakayama Prefecture and transported to NRIA as a parasite source. Worm eggs were collected as previously described (Yoshinaga et al., 2001). 
Fifty flounder (12-16 cm in total length) were maintained in each of two 150-L tanks. In one tank, a nylon mesh bag of $108 \mu \mathrm{m}$ mesh opening containing 5000 worm eggs was suspended for the challenge. In the other tank, as a control, a nylon mesh bag without any worm eggs was suspended. On Day 56 post challenge, the challenged fish were divided into two 150-L tanks, each containing 25 and 24 fish (one fish escaped from the tank and was found dead after the challenge). Twenty-five of the control fish were transferred to one tank. All the fish were individually marked with plastic tags and bled by dorsal aorta puncture for hematological examination. Twenty-five of the challenged fish were treated to remove the worms as follows; adult worms on the buccal cavity wall were removed by forceps and immature worms on the gills were removed by $3 \% \mathrm{NaCl}-$ supplemented seawater bathing for 60 min according to Yoshinaga et al. (2000b). Removed adult worms were counted. The control fish and the rest of the challenged fish were maintained without treatment. On Day 77, all fish were bled by dorsal aorta puncture for hematological examination and sacrificed for parasitological examination. During the experiment, the fish were kept at $15-16^{\circ} \mathrm{C}$ and fed commercial pellets three times a week. The water turnover rates were 10-20/day until Day 56 and 40-50/day from Day 56 until the end of the experiment.

In the hematological examination, $\mathrm{Hb}$ of blood was determined using the cianmethohemoglobin method and the blood smear was stained with the May-GrünwaldGiemza stain. Based on the microscopic observations of each blood smear, fish were given a blood smear type and a scored ratio of immature erythrocytes as follows according to Yoshinaga et al. (2001). Blood smear types 1,2, and 3 represent fish without abnormality in erythrocytes, with abnormality only in immature erythrocytes, with abnormality in both immature and mature erythrocytes, respectively. Scored ratios of immature erythrocytes $0,1,2,3$ and 4 represent the ratios of the number of immature erythrocytes to the total number of erythrocytes below 5\%, 5-25\%, 25-50\%, 50-75\%, and over $75 \%$, respectively. In the parasitological examination, adult worms on the buccal cavity wall were removed and macroscopically counted. Immature worms on the gills were counted according to Yoshinaga et al. (2001).

\section{Results}

Neoheterobothrium hirame infections in the experimental groups on Day 56 and 77 are shown in Table 1. In the control group, none of fish was infected with $N$. hirame. In the treated group, 24 out of the 25 fish were infected with adult worms on the buccal cavity wall on Day 56. On Day 77, none of the treated fish was infected, indicating the treatment was successful. The prevalence of adult worms in the untreated group on Day
77 was lower than that in the treated group on Day 56; 14 out of the 24 fish were infected in the untreated group. No immature worms were obtained from the gills of both the treated and untreated fish.

Anemia with a low $\mathrm{Hb}$ and abnormal erythrocytes having a vacuolated or weakly stained cytoplasm was induced in the challenged fish on Day 56 (Figs. 1 and 2), although abnormal mature erythrocytes were observed only in one out of the 49 challenged fish. Abnormal erythrocytes appeared in fish with a $\mathrm{Hb}$ lower than ca. 3

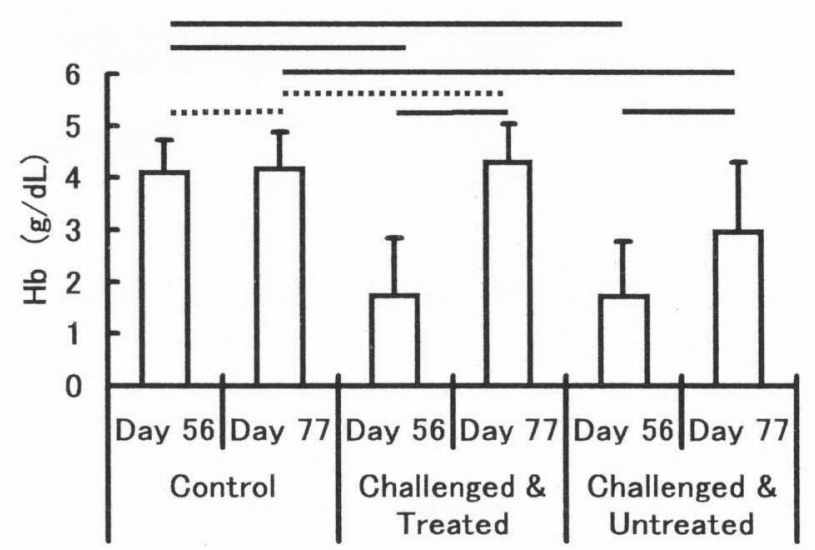

Fig. 1. Changes in the mean hemoglobin concentration $(\mathrm{Hb})$ in the experimental groups. Vertical bars represent SD. Solid and dotted horizontal lines represent a highly significant difference $(P<0.01)$ and an insignificant difference ( $P>0.05)$ between groups at the ends of the lines (Mann-Whitney's U-test), respectively.

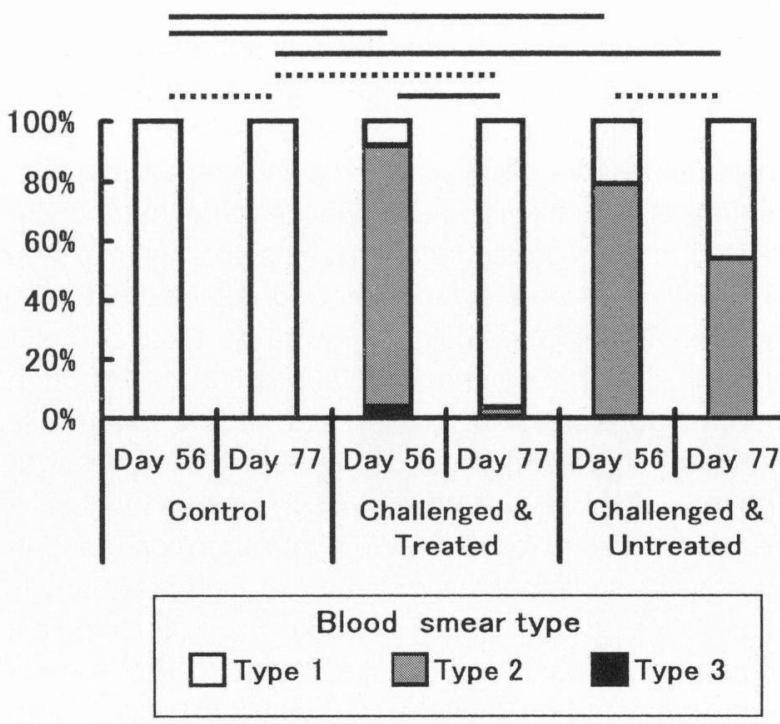

Fig. 2. Changes in the frequency of blood smear types in the experimental groups. Solid and dotted horizontal lines represent a highly significant difference $(P<0.01)$ and an insignificant difference $(P>0.05)$ between groups at the ends of the lines (Fisher's exact probability test), respectively. For the statistical analysis, the numbers of flounder with blood smear types 2 and 3 were combined. 
$\mathrm{g} / \mathrm{dL}$, and the ratio of the immature erythrocytes increased with decreasing $\mathrm{Hb}$ (Fig. 3). The $\mathrm{Hb}$ showed a negative correlation with the number of adult worms in the treated group (Fig. 4).

In the treated group, the mean $\mathrm{Hb}$ significantly increased to the level similar to that in the control group and fish having abnormal erythrocytes disappeared except one individual out of 24 fish by Day 77 , three

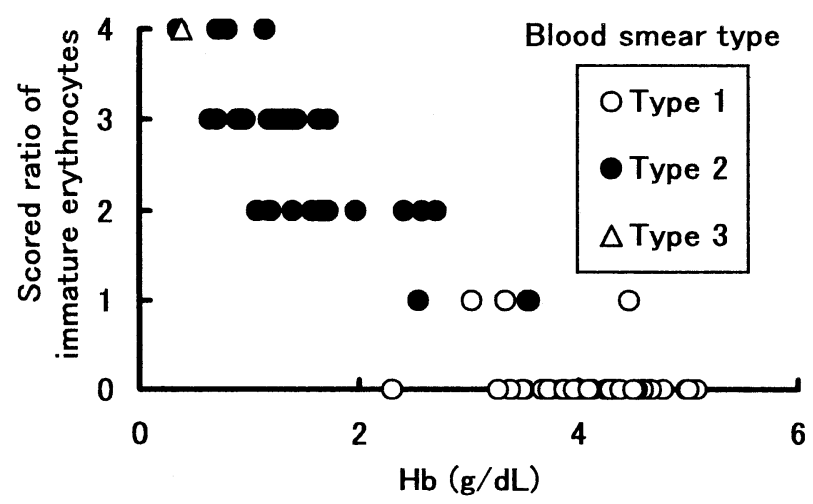

Fig. 3. Relationship between hemoglobin concentration $(\mathrm{Hb})$, scored ratios of immature erythrocytes and blood smear types in both the challenged and control flounder on Day 56.

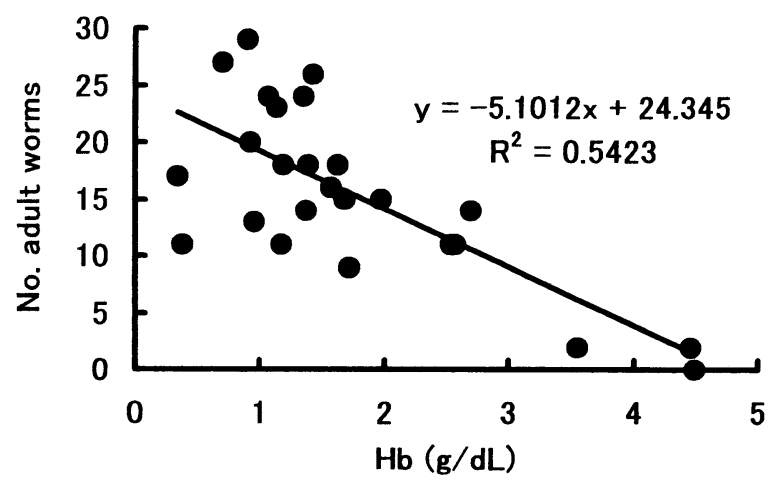

Fig. 4. Relationship between hemoglobin concentration ( $\mathrm{Hb})$ and the number of adult Neoheterobothrium hirame in the treated group on Day 56. Regression formula and correlation coefficient are given. weeks after the worm removal (Figs. 1 and 2). Whilst, in the untreated group, the mean $\mathrm{Hb}$ was still significantly lower than that in the control group on Day 77 , although it slightly increased between Day 56 and Day 77. Moreover, abnormal erythrocytes were observed in as many as 13 out of 24 fish on Day 77.

\section{Discussion}

Yoshinaga et al. (2001) demonstrated that low $\mathrm{Hb}$ and abnormal immature and mature erythrocytes having a vacuolated or weekly stained cytoplasm, which characterize the recent anemia in wild and cultured Japanese flounder, were induced in Japanese flounder by the challenges with Neoheterobothrium hirame and a negative correlation was observed between $\mathrm{Hb}$ and the number of adult worms in the challenged flounder. Also in the present study, similar results were obtained by the challenge with the parasite. Although abnormal mature erythrocytes were observed only in one fish in the present study, it is probably because the fish were fed during the experiment, considering the result of Yoshinaga et al. (2001) that the starved fish showed severer anemic conditions than the fed fish in their challenge experiments with the parasite.

When $N$. hirame were removed from the anemic flounder generated by the parasite challenge, their hematological conditions clearly improved; the $\mathrm{Hb}$ increased to the levels observed in the control group, and fish having abnormal erythrocytes almost disappeared. Although the $\mathrm{Hb}$ increased to some extent also in the untreated group, the increase was much lower than that in the treated group. The increase in $\mathrm{Hb}$ in the untreated group seemed to be caused by natural detachment of $N$. hirame between Day 56 and Day 77 , based on the observation that the prevalence of the parasite in the untreated group on Day 77 was lower than that in the treated group on Day 56 (Table 1). Such natural detachments of $N$. hirame adults were also observed previously in both naturally and experimentally infected flounder (Yoshinaga et al., 2000a, 2001).

Table 1. Neoheterobothrium hirame infection in experimental groups of Japanese flounder

\begin{tabular}{lcccc}
\hline & & \multicolumn{2}{c}{ Adult worm } & Immature worm \\
\cline { 3 - 5 } & $\begin{array}{c}\text { Days post } \\
\text { challenge }\end{array}$ & $\begin{array}{c}\text { Prevalence } \\
\text { (no. infected/total) }\end{array}$ & $\begin{array}{c}\text { Intensity*1 } \\
\text { Mean (range) }\end{array}$ & $\begin{array}{c}\text { Prevalence } \\
\text { (no. infected/total) }\end{array}$ \\
\hline Control & 56 & $0 / 25$ & 0 & NE*2 \\
& 77 & $0 / 25$ & 0 & 0 \\
Challenged \& & 56 & $24 / 25$ & $16.2(2-29)$ & NE \\
Treated & 77 & $0 / 25$ & 0 & 0 \\
Challenged \& & 56 & $\mathrm{NE}$ & $\mathrm{NE}$ & $\mathrm{NE}$ \\
Untreated & 77 & $14 / 24$ & $9(2-17)$ & 0 \\
\hline$* 1$ No. of worms on infected fish. & & & \\
$* 2$ & & &
\end{tabular}


Miwa and Inouye (1999) suggested that $N$. hirame might be a vector of some causative virus of the recent anemia in Japanese flounder. However, in this study, the anemic flounder generated by the challenge with the parasite were successfully cured by the artificial removal of the parasite. It is, therefore, clear that the anemia was caused by $N$. hirame itself. If some etiological agent other than $N$. hirame had caused the anemia and the parasite had acted as the vector of the agent, the anemia would not have been cured by the mere removal of the parasite. To the best of our knowledge, there have been no reports so far demonstrating that monogeneans can act as vectors of some viruses. Moreover, no special organ needed to inject viruses into the host has been described for $N$. hirame (see Ogawa, 1999). These also indicate that $N$. hirame is not a vector of any causative viruses. The present results support our previous suggestion that the anemia recently prevailing in wild and cultured Japanese flounder is caused by the blood-feeding activity of $N$. hirame (Yoshinaga et al., 2000a, 2001). Furthermore, from the present result that the anemic condition disappeared in three weeks after the removal of the parasite, it is indicated that the removal of the parasite is effective as a treatment of the anemia.

\section{Acknowledgements}

We thank Mr. Terufumi Takeuchi, Wakayama Prefectural Fisheries Farming Experimental Station, for pro- viding us with wild flounder infected with $N$. hirame. We also thank Ms. Junko Hiraga, National Research Institute of Aquaculture, for her technical assistance. This study was supported by a grant-in-aid from Ministry of Agriculture, Forestry and Fisheries, Japan.

\section{References}

Michine, A. (1999): Neoheterobothrium sp. found on Japanese flounder cultured commercially or maintained as spawners. Res. Shimane Pref. Center Cult. Fish., 2, 15-23. (In Japanese)

Miwa, S. and K. Inouye (1999): Histopathological study of the flounder with anemia found in various places in Japanese coastal waters. Fish Pathol., 34, 113-119. (In Japanese with an English abstract)

Ogawa, K. (1999): Neoheterobothrium hirame sp. nov. (Monogenea: Diclidophoridae) from the buccal cavity wall of Japanese flounder Paralichthys olivaceus. Fish Pathol., 34, 195-201.

Yoshinaga, T., T. Kamaishi, I. Segawa, A. Kumagai, C. Nakayasu, T. Takeuchi and M. Sorimachi (2000a): Hematology, histopathology and the monogenean Neoheterobothrium hirame infection in anemic flounder. Fish Pathol., 35, 131-136. (In Japanese with an English abstract)

Yoshinaga, T., T. Kamaishi, I. Segawa and E. Yamamoto (2000b): Effects of $\mathrm{NaCl}$-supplemented seawater on the monogenean, Neoheterobothrium hirame, infecting the Japanese flounder. Fish Pathol., 35, 97-98.

Yoshinaga, T., T. Kamaishi, I. Segawa, K. Yamano, H. Ikeda and M. Sorimachi (2001): Anemia caused by challenges with the monogenean, Neoheterobothrium hirame in Japanese flounder. Fish Pathol., 36, 13-20. 